\title{
1 Effect of large diameter and plasma coating on the initial adaptation of gas \\ 2 permeable contact lens fitting for neophytes
}

3

4 Authors: Dutta, Debarun; Wolffsohn, James S.

5 Optometry and Vision Science Research Group, Optometry School, Aston University, UK

6

7 Running title: Initial GP lens adaptation with large diameter and plasma coating for neophytes

8

9 Corresponding author: James Wolffsohn, Aston University, Birmingham, B4 7ET, UK;

$10 \quad$ J.S.W.Wolffsohn@aston.ac.uk

11

Conflict of interest: This work is original, has not been published and is not being considered for publication elsewhere. The authors disclose no commercial relationship associated with this research.

Acknowledgement: The GPs were provided and coated by No7 Contact Lenses and Contamac. The research was part funded by Innovate UK, Technology Strategy Board Grant (102544)

Key words: Rigid gas permeable (GP), contact lens, adaption, diameter, plasma coating, ocular 18 comfort, neophytes

\section{Transparency Declaration}

The corresponding author affirms that this manuscript is an honest, accurate, and transparent account of the study being reported; that no important aspects of the study have been omitted; and there is no discrepancy from the study as planned.

\section{Abstract}

Purpose: To determine whether the adaption of neophytes to rigid gas permeable lenses (RGPs) could be accelerated by changing their diameter or surface lubricity.

Methods: This was a 2 part prospective double-masked randomised bilateral wear study. An RGP lens (Roflufocon D material, 9.6mm diameter) with and without a Hydrapeg plasma coating surface was worn by 119 neophytes ( $21.1 \pm 3.5$ years; $77 \%$ female) on separate occasions. A further 114 neophytes (average age $20.1 \pm 1.0$ years; $72 \%$ female) wore the uncoated lens with a randomly allocated $9.6 \mathrm{~mm}$ diameter lens one one eye and $10.1 \mathrm{~mm}(n=51)$ or $10.6 \mathrm{~mm}(n=63)$ in the other with a basecurve compensation keeping the fit the same. Lens fit and corneal staining was assessed after 20 minutes, and comfort and bulbar redness were assessed at the time of lens application and after 
$345,10,15$, and 20 minutes. Participants also reported their ease of application and removal on a 5 35 point scale.

36 Results: Plasma coating RGP lenses or increasing their diameter did not improve comfort $(p=0.673$, $37 p>0.05)$ or bulbar redness $(p=0.805, p>0.05)$ during a 20 minute adaptation period in neophytes. In 38 both cohorts, comfort improved and bulbar redness reduced with time $(p<0.001)$. Corneal staining, 39 ease of insertion application and ease of removal did not differ with RGP coating application or RGP 40 diameter $(p>0.05)$.

41 Conclusion: Changing RGP diameter or surface lubricity is not beneficial to the adaption of 42 neophytes 
Introduction:

Rigid Gas Permeable (GP) contact lenses occupy $13 \%$ of global contact lens fits, with $10 \%$ fits for conventional GP lenses and 3\% with Orthokeratology (OK) lenses.[1] Based on the area and location, conventional GP lens fits account from 5\% (United Kingdom) to 27\% (France and Netherlands) of all lens fits.[1] Although the wearing schedule and the type of contact lenses available in the market have changed substantially over the last couple of decades, the global share of GP lens fits over all types of lenses remained steady between $10 \%$ to $20 \%$.

The benefits of GP lenses are well known; superior ocular hygiene and health, quality of vision, surface wettability, high oxygen transmission, reduction of myopia progression (through orthokeratology), reduced incidence and severity of corneal infiltrative events to name a few. $[2,3]$ The primary concern for the GP lens wear is that 'Rigid' lenses are uncomfortable and often painful, particularly for the initial adaptation period. $[4,5]$. This adaptation period varies between studies, with successful wearers achieving good comfort and subjective vision within 10-15 days in one study [6], but $23 \pm 22.1$ days in another [7].

Thus the question arises as to how the initial problem with comfort and epiphora can be minimised to reduce fear within patients and enhance GP lens use? One approach that has been explored has been the use of topical anaesthetic at the time of initial fitting and possibly at the time of lens dispensing. Bennett et al.[8] investigated this effect on 80 participants and concluded that use of topical anaesthesia enhanced perception for initial adaptation process, greater satisfaction after one-month lens wear and significantly reduced drop out compared to participants who did not receive topical anaesthesia[8]. However, a survey of UK eye care practitioners ( $n=451)$ found less than $1.5 \%$ used anaesthetic regularly and less than one-third (30.3\%) considered it clinically acceptable to do so [9]. Alteration of certain lens designs has also been investigated while tackling this issue. The use of well-blended lens, smoothening of the edge and optimum cornea-lens fitting 
68 relationships are recommended to enhance initial comfort[10]. There is some evidence that larger

69 GPs are more comfortable for adapted wearers [11], but no research has examined the adaptation 70 stage.

71 Plasma treatment such as oxygen plasma for GP lens surfaces has been around for many years, and 72 often used for cleaning and removing any remaining residues from the lens manufacturing process.

73 The idea behind this treatment is to enhance the wetting angle, resulting in a more hydrophilic lens

74 surface.[12] Applying a plasma coating to soft contact lenses has been shown to enhance lens

75 surface lubricity, which appears to be the principal contributor to comfort.[13]However, its effect on

76 GP lens comfort has not been investigated.

77 The purpose of this current study was to determine whether a plasma coating or fitting a lens with a 78 larger diameter can make GP lenses more comfortable to patients during initial adaptation to first 79 time wear and have less impact to ocular surface physiology. 
82 This was a prospective double-masked randomised study which was conducted according to Good

83 Clinical Practice Guidelines. Individuals within the University student and faculty community with

84 healthy eyes, no previous ocular surgery and no history of rigid contact lens wear were recruited to

85 participate in this study. This study received approval from the Aston University human research

86 ethics committee and adhered to the tenets of the declaration of Helsinki (2013). Two different

87 cohorts of participants were recruited for investigation of the effect of larger diameter and plasma

88 coating of GP lenses (Figure 1).
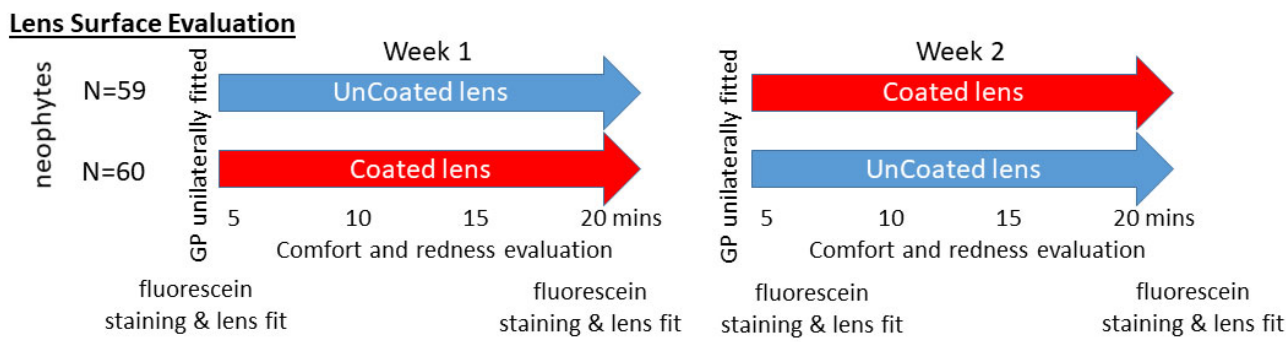

$\underline{\text { Lens Diameter Evaluation }}$

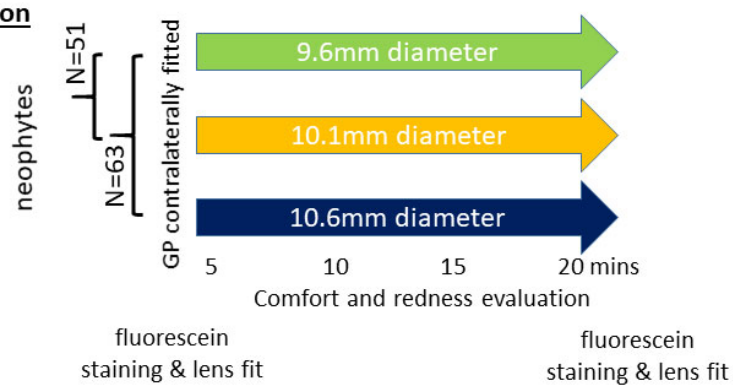

89

Figure 1. Flow diagram of study cohorts

91

92 A total of 119 participants were recruited for investigating the impact of plasma coating on the initial

93 comfort and adaptation. Detailed eye examinations related to contact lens wear was conducted

94 during two study visits, scheduled at the one-week interval and at the same time as the day \pm 2 hours.

95 GP lenses (Roflufocon D material, 9.6mm diameter; No7 Contact Lenses, Hastings, UK) made of the same design with and without a Hydrapeg plasma coating (Contamac, Safron Waldon, UK) surface 
were used for this study. Polyethylene glycol (PEG) has been used in ocular lubricants for decades and is known to improve lens surface wettability and lubricity, reducing friction between the lens and the eyelids.[13, 14] Tangible Hydra-PEG is a $90 \%$ water PEG-based polymer mixture that is covalently bonded to the surface of the contact lens, creating a wetting surface overlying the lens material. Lens base curve was determined by the average keratometry readings for each individual participant.

A total of 114 participants were recruited for the assessment of large diameter GP lens. Fifty one participants were randomly allocated to wear an uncoated (Roflufocon D material) $9.6 \mathrm{~mm}$ diameter GP lenses in one eye and a $10.1 \mathrm{~mm}$ lens in the other, and another 63 participants were allocated to also wear a $9.60 \mathrm{~mm}$ diameter lens in one eye, but a $10.6 \mathrm{~mm}$ GP lenses in the other. Lens base curve was determined by the average keratometry readings for each individual participant, compensated according to the prescribed diameter so that changes in the lens diameter did not affect the cornea-lens interaction and ocular physiology.

In both studies, comprehensive assessment of the GP contact lens fitting was conducted at each visit including lens fit 1-3 minutes after fluorescein instillation, $[15,16]$ assessment of bulbal redness to the nearest 0.1 unit step using Johnson and Johnsons (J\&J) clinical grading scale, $[17,18]$ and subjective assessment of contact lens wear comfort using the visual analogue scale (VAS) at the time of lens application and after 5, 10, 15, and 20 minutes. Corneal fluorescein staining was graded at the time of lens application and after 20 minutes of wear. Participants also reported their ease of application and removal on a 5 point scale.

\section{Statistical analysis:}

All data were analysed using SPSS (version 26; IBM, Chicago,II, USA). As the data differed from a normal distribution as determined using the Kolmogorov-Smirnov test, non-parametric statistics were applied. Wilcoxon Signed-Rank test was used to determine significant difference for non- 

of $\leq 0.05$ was considered statistically significant.

123 
Results:

125 The mean age for the 119 participants for the plasma coated GP lens trial was $21.1 \pm 3.5$ years, among

126 them $77 \%$ were female. The mean age for the 114 participants for large diameter GP lens trial was

$12720.1 \pm 1.0$ years and $72 \%$ of them were female. There were no drop outs in this study, none of the

128 participants developed any complication during contact lens wear.

129 Effect of plasma coating on initial GP lens fitting:

130 Lens fit was not affected by the lens coating ( $p=0.972$ ). Plasma coated GP lenses had a negligible

131 effect on enhancing initial discomfort when compared to uncoated GP lenses ( $p=0.673$; Figure 2$)$.

132 Ocular comfort was $91.4 \pm 13.2$ and $93.9 \pm 10.2$ prior to lens wear, which dropped to $28.8 \pm 26.2$ and

$13325.5 \pm 22.3$ immediately after, and slightly improved to $53.7 \pm 21.4$ and $55.0 \pm 22.0$ after 20 minutes of

134 uncoated and coated GP lens wear respectively. Comfort with both coated and uncoated GP lenses

135 increased significantly with time after application $(p<0.001)$.

\section{Comfort}

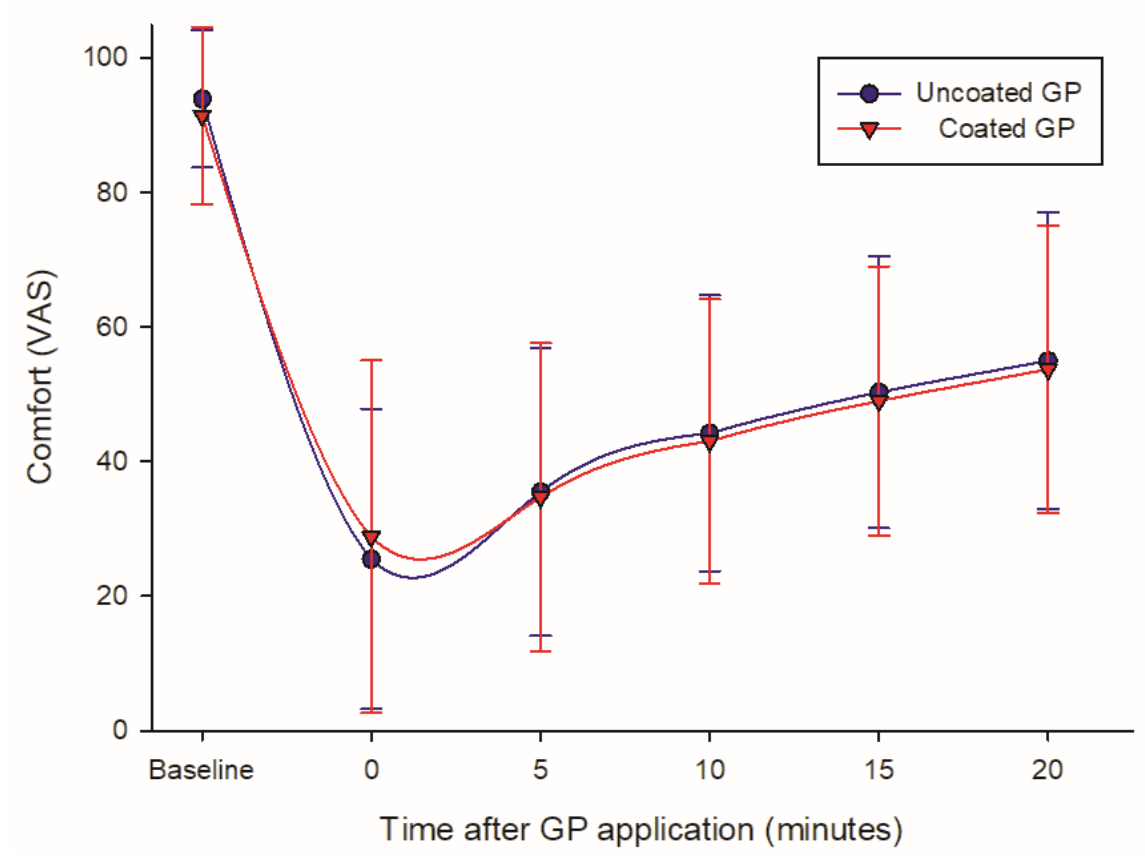

Figure 2. Effect of plasma coated GP lens wear on initial comfort determined by VAS. 

immediately after lens wear, and the difference with non-coated GP lens wear was not statistically significant ( $p=0.805$; Figure 3 ). Bulbar redness was $1.3 \pm 0.5$ and $1.2 \pm 0.5$ prior to lens wear, which increased to $1.7 \pm 0.7$ and $1.7 \pm 0.7$ immediately after, followed by slight drop to $1.5 \pm 0.6$ and $1.5 \pm 0.6$ after 20 minutes of uncoated and coated GP lens wear respectively. The drop in bulbar redness following coated and uncoated lens wear after 20 minutes was statistically significant $(p<0.001)$. In addition, corneal staining $(p=0.819)$, ease of application $(p=0.729)$ and ease of removal $(p=0.806)$ did not differ with GP coated lens wear.

\section{Bulbar Redness}

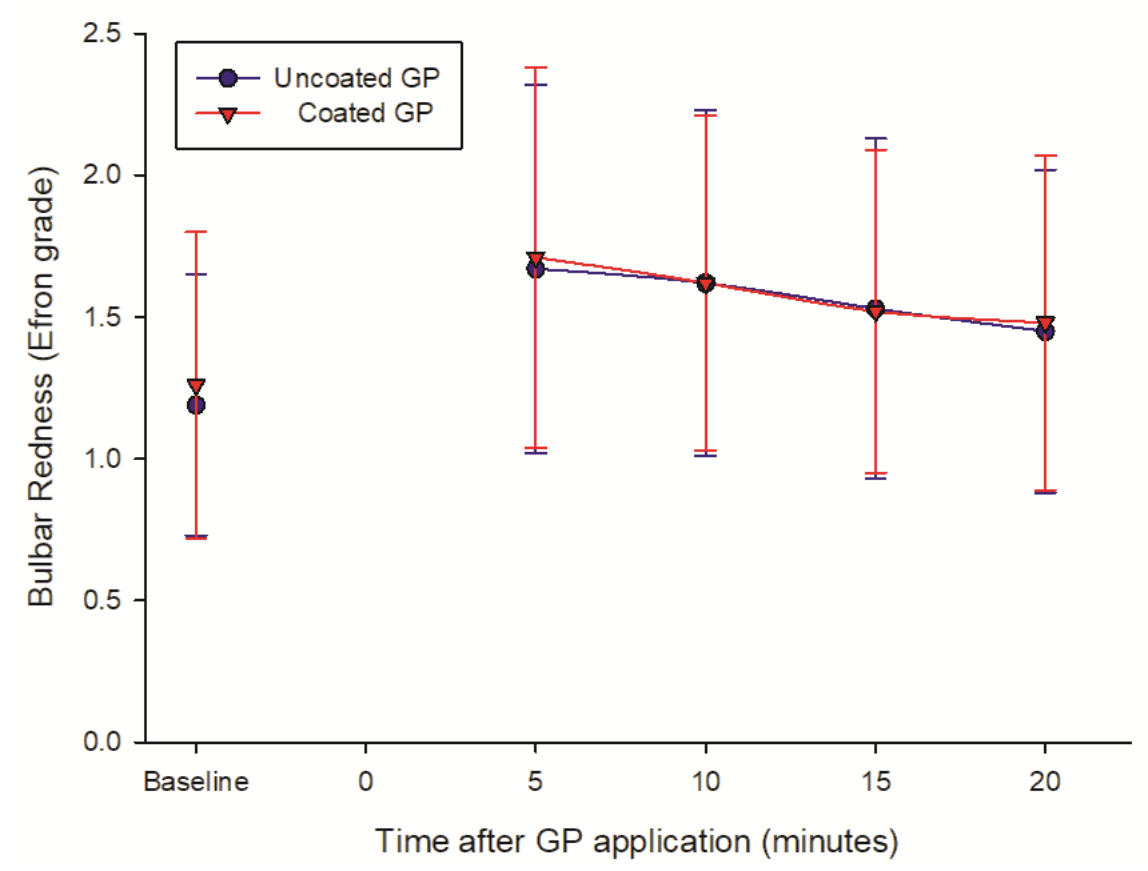

Figure 3. Effect of plasma coated lens wear on bulbar redness after lens application.

Effect of large diameter GP lens fitting:

151 Lens fit was similar between the GP diameters assessed $(p=0.417)$, due to the base-curve

152 compensation to ensure this did not affect the comfort or ocular physiology.

153 Comfort changed with time $(p<0.001)$, decreasing on GP application and improving every 5 minutes 154 ( $p<0.005)$ except for between 15 and 20 minutes for the 9.6 and $10.1 \mathrm{~mm}$ GP diameters (Figure 4). 

the different sizes of GP lens wear ( $p>0.05)$.

\section{Comfort}

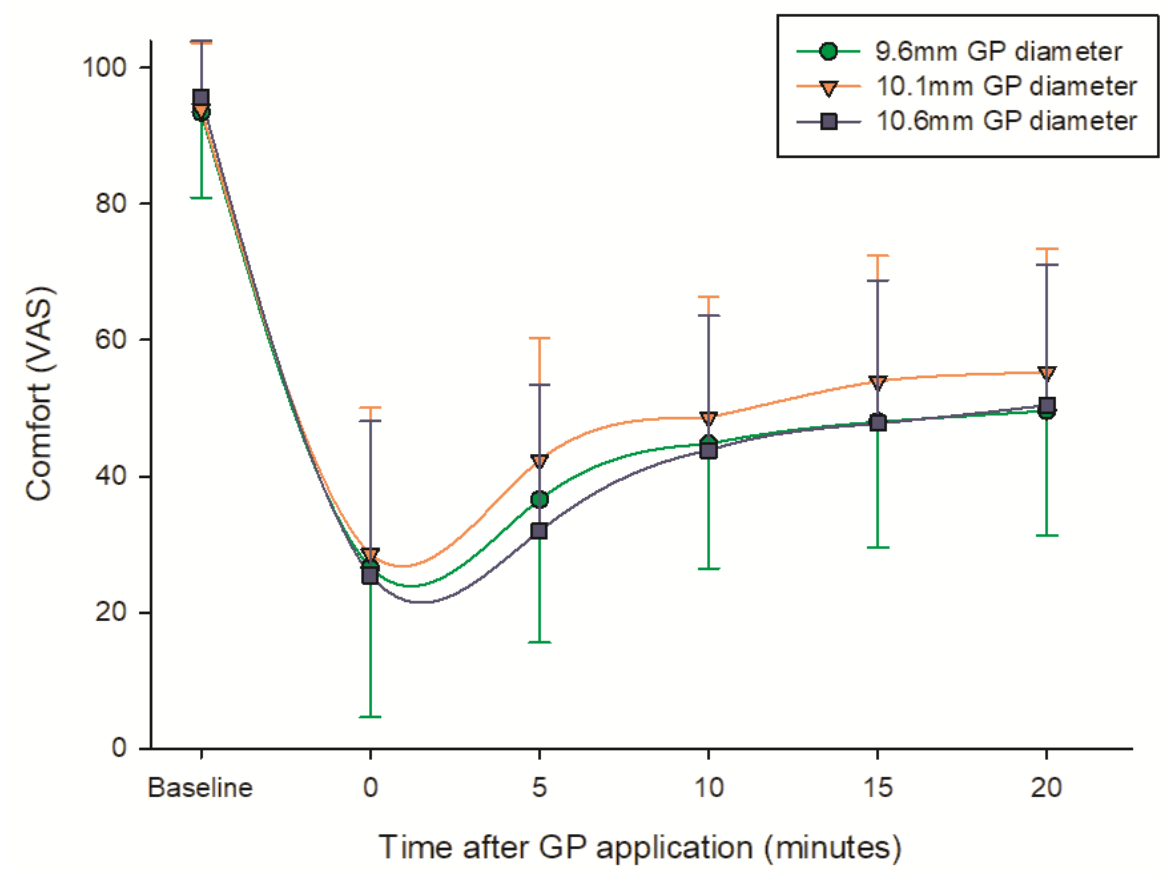

Figure 4. Ocular comfort following large-diameter GP lens wear determined by visual analogue scale. between 5 minute time intervals and was still significantly lower than baseline 20 minutes after GP application ( $p<0.001$; Figure 5$)$. There was no difference at any time point between the different sizes of GP lens wear ( $p>0.05)$. In addition, corneal staining $(p=0.368)$, ease of application $(p=0.419)$ and ease of removal $(p=0.274)$ did not differ with varying GP diameter. 


\section{Bulbar Redness}

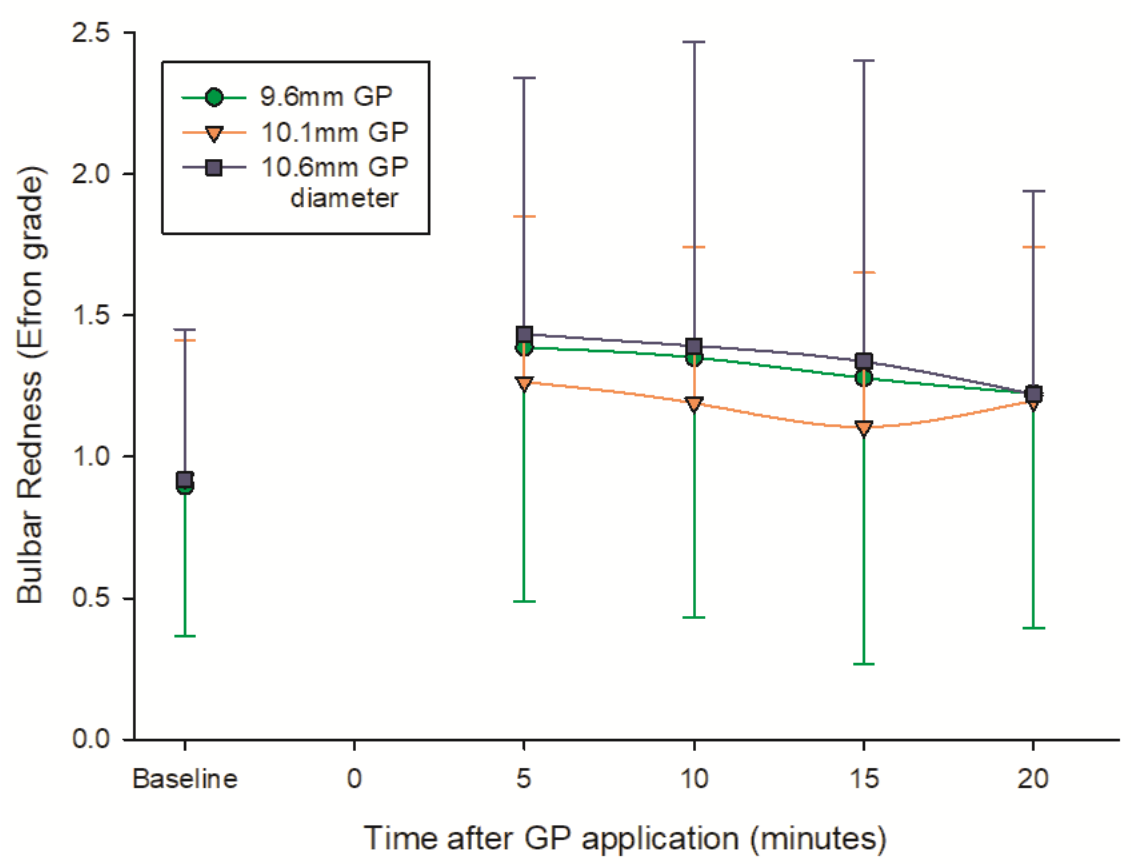

167

Figure 5. Impact of large diameter GP lens wear on bulbar redness

168

169 
171 The current study, for the first time, investigated the effect of lens surface enhancement and diameter on the initial comfort and adaptation to GP lens fitting by neophytes. The current study showed that having a plasma coating or increasing the diameter may not alleviate immediate signs and symptoms related to initial adaptation to GP lens wear, at least for the initial 20 minutes.

Various types of plasma treatments are being used for modification of biomaterial surfaces, particularly for modifying a hydrophobic surface to a more hydrophilic state.[19] Hydrophilic surfaces are known for high biocompatibility, reduced inflammation and friction with surrounding tissues. As an example, surface modified hydrophilic intraocular lenses have been associated with increased uveal biocompatibility.[20] PEG is widely used as a modification molecule which is grafted on a surface to repel proteins due to its high dipole moment that provides enhanced hydration. The application of plasma treatment to enhance the surface wettability of first generation silicone hydrogel contact lenses has been widespread.[21] The Lotrafilcon A material uses a reactive plasma process to precipitate a thin layer hydrophilic polymer,[22] whereas Balafilcon A is subject to plasma oxidation process masking its hydrophobic core with increased surface wettability.[21] Similarly this study used Hydrapeg surface coating onto conventional GP lens material which is known to increase surface wettability.[23]

The results of this study showed that surface treatment had little or no effect on the initial signs and symptoms of GP lens wear for neophytes. The reduction of comfort observed immediately after lens insertion with the plasma coated lenses was similar to the observation with uncoated lenses. Following this, the gradual increase observed with lens wear was similar in both types of lenses. Similar results were observed with ocular redness, where the bulbar redness increased following GP lens wear and slowly decreased over time for both plasma coated and uncoated GP lenses. The finding would suggest that it is not friction with the lens surface that drives initial discomfort with GP wear, rather the bulk of the lens and its interaction with the eyelids and ocular surface. Visual 
analogue discomfort scores [7] and the Pain Sensitivity Questionnaire [24] have been found to help predict those who will successfully adapt to GP wear, whereas lid sensitivity did not.

Hence, the effect of lens diameter was investigated in a separate cohort, increasing from a more standard $9.6 \mathrm{~mm}$ lens to a $10.1 \mathrm{~mm}$ or $10.6 \mathrm{~mm}$ corneal GP lenses for assessment of initial adaptation and comfort. Although this did not affect the cornea-lens fitting characteristics, larger diameter lenses had a negligible impact on the initial adaptation, signs and symptoms for neophyte participants. Both ocular signs and symptoms for the $10.1 \mathrm{~mm}$ and $10.6 \mathrm{~mm}$ GP lenses were comparable with standard $9.6 \mathrm{~mm}$ GP lens wear. There was no significant difference in bulbar redness following large diameter GP lens wear compared to standard diameter $(9.6 \mathrm{~mm})$ GP lenses. In contrast, a previous study by Gispets et al. with nine patients showed that larger diameter GP lenses were associated with increased ocular comfort after 10 minutes, but there is limited information in this conference abstract.[25] Williams-Lyn et al. found that a $10.0 \mathrm{~mm}$ lens was significantly more comfortable than $9.0 \mathrm{~mm}$ and $9.5 \mathrm{~mm}$ diameter lenses; [26] however, the study only involved 12 participants, all of whom were experienced GP lens wearers, whereas the current study recruited only neophytes for the assessment of initial adaptation. Other studies have used corneo-scleral or mini-scleral large diameter GP lenses.[27, 28] Most of these lenses ranged between 13 to $16 \mathrm{~mm}$ in diameter that resulted in reduced ocular redness and enhanced comfort. However, these lenses rest on conjunctiva bypassing any major interaction with cornea which significantly reduce lid-lens interaction; hence comparison with their results with the current study is of limited 214 value. The current study was conducted on participants with healthy eyes who may not be motivated for long-term GP lens wear, which is different to patients who have had penetrating keratoplasty or radial keratotomy, or patients with keratoconus or high irregular astigmatism.[29, 30] In addition, this study aimed to investigate initial adaption over the first 20 minutes of lens wear; perhaps longer observation may be necessary to understand the extended impact of plasma coating and lens 
220 diameter. Lastly, the participants were young health adults who may be more symptomatic to GP

221 lens wear compared to an older population or individuals with underlying corneal conditions.

222 In conclusion, the outcome of this study provides valuable information for contact lens practitioners

223 that additional plasma coating or larger diameter corneal GP lenses may not be a viable option to

224 mitigate the initial discomfort and adaptation problems related to first time GP lens wear. Clinical

225 investigations with longer follow up durations are required for assessment of their long term effect

226 on ocular signs and symptoms. 
229 [1] P. Morgan, C.A. Woods, Tranoudis, N. Efron, L. Merchan, Grupcheva., D. Jones, M. Beeler-Kaupke, 230 Krasnaska., S. Belova, Santodomingo-Rubido., P. Taste, Malet., Plakitsi., Vegh., Erdinest., G. Montani, Romualdez-Oo., Abesamis-Dichoso., J.M. González-Méijome, Gierow., Pettersson., Hsiao., J.J. Nichols, International Contact Lens Prescribing in 2020, Contact Lens Spectrun 2020, pp. 26-32. [2] K.A. Polse, A.D. Graham, R.E. Fusaro, C.M. Gan, R.K. Rivera, J.S. Chan, N.A. McNamara, T.S. Sanders, Predicting RGP daily wear success, The CLAO journal : official publication of the Contact Lens Association of Ophthalmologists, Inc 25(3) (1999) 152-8.

[3] K.A. Polse, A.D. Graham, R.E. Fusaro, C.M. Gan, R.K. Rivera, M.C. Lin, T.L. Sanders, N.A. McNamara, J.S. Chan, The Berkeley Contact Lens Extended Wear Study. Part II : Clinical results, Ophthalmology 108(8) (2001) 1389-99.

[4] R. Martin, E. Alonso, Comparison of the number of visits and diagnostic lenses required to fit RGP, conventional hydrogel and silicone hydrogel contact lenses, Journal of Optometry 3(3) (2010) 169-174.

[5] S.J. Vincent, The rigid lens renaissance: A surge in sclerals, Cont Lens Anterior Eye 41(2) (2018) 139-143.

[6] G. Carracedo, A. Martin-Gil, S.C. Peixoto-de-Matos, P. Abejon-Gil, R. Macedo-de-Araujo, J.M. Gonzalez-Meijome, Symptoms and signs in rigid gas permeable lens wearers during adaptation period, Eye and Contact Lens 42 (2016) 108-114.

[7] H. Fujita, K. Sano, S. Sasaki, K. Ohno-Matsui, T. Tanaka, T. Baba, M. Mochizuki, Ocular discomfort at the initial wearing of rigid gas permeable contact lenses, Japanese journal of ophthalmology 48(4) (2004) 376-9.

[8] E.S. Bennett, J. Smythe, V.A. Henry, C.J. Bassi, B.W. Morgan, W. Miller, M. Jeandervin, B. Henderson, L. Elliott, K.S. Porter, J.T. Barr, Effect of topical anesthetic use on initial patient satisfaction and overall success with rigid gas permeable contact lenses, Optom Vis Sci 75(11) (1998) 800-5.

[9] F.R. Gill, P.J. Murphy, C. Purslow, A survey of UK practitioner attitudes to the fitting of rigid gas permeable lenses, Ophthalmic Physiol Opt 30(6) (2010) 731-9.

[10] E. van der Worp, J. de Brabander, B. Lubberman, G. Marin, F. Hendrikse, Optimising RGP lens fitting in normal eyes using 3D topographic data, Cont Lens Anterior Eye 25(2) (2002) 95-9. [11] S. Williams-Lyn, K. MacNeill, D. Fonn, The effect of RGP back optic zone radius and diameter changes on comfort, International Contact Lens Clinic 20 (1993) 223-118.

[12] M. Bergmann, L. Ledernez, G. Dame, S. Lickert, F. Widmer, Y. Gier, G. Urban, Nanofilms Produced by Magnetron Enhanced Plasma Polymerization from Methane and Oxygen for Coating of Rigid Contact Lenses, Plasma Processes and Polymers 10(11) (2013) 970-977.

[13] M. Vidal-Rohr, J.S. Wolffsohn, L.N. Davies, A. Cervino, Effect of contact lens surface properties on comfort, tear stability and ocular physiology, Cont Lens Anterior Eye 41(1) (2018) 117-121. [14] L. Jones, N.A. Brennan, J. Gonzalez-Meijome, J. Lally, C. Maldonado-Codina, T.A. Schmidt, L. Subbaraman, G. Young, J.J. Nichols, T.I.W.o.C.L.D. members of the, The TFOS International Workshop on Contact Lens Discomfort: report of the contact lens materials, design, and care subcommittee, Invest Ophthalmol Vis Sci 54(11) (2013) TFOS37-70.

[15] J.S. Wolffsohn, E. van der Worp, J. de Brabander, G.P.C. Group, Consensus on recording of gas permeable contact lens fit, Cont Lens Anterior Eye 36(6) (2013) 299-303.

[16] J.S. Wolffsohn, A. Tharoo, N. Lakhlani, Optimal time following fluorescein instillation to evaluate rigid gas permeable contact lens fit, Cont Lens Anterior Eye 38(2) (2015) 110-4.

[17] N. Efron, Grading scales for contact lens complications, Ophthalmic Physiol Opt 18(2) (1998) $182-6$.

[18] N. Efron, Clinical application of grading scales for contact lens complications, Optician 213(5606) (1997) 26-35. 
279 Udipi, Impact of polymer hydrophilicity on biocompatibility: implication for DES polymer design, J

280 Biomed Mater Res A 90(1) (2009) 133-41.

281 [20] Q. Huang, G.P. Cheng, K. Chiu, G.Q. Wang, Surface Modification of Intraocular Lenses, Chin Med 282 J (Engl) 129(2) (2016) 206-14.

283 [21] N.A. Brennan, M.L. Coles, T.L. Comstock, B. Levy, A 1-year prospective clinical trial of balafilcon a (PureVision) silicone-hydrogel contact lenses used on a 30-day continuous wear schedule, Ophthalmology 109(6) (2002) 1172-7.

[22] G. Young, R. Garofalo, O. Harmer, S. Peters, The effect of soft contact lens care products on lens modulus, Cont Lens Anterior Eye 33(5) (2010) 210-4.

[23] C.W. Sindt, Tangible Hydra-PEG: A novel custom contact lens coating technology designed to improve patient comfort and satisfaction, Whitepaper, Blanchard, 2016. [24] D.S. Nosch, R.E. Joos, D. Müller, S.M. Matt, General pain perception sensitivity, lid margin sensitivity and gas permeable contact lens comfort, Clin Exp Optom (2019). [25] J. Gispets, V. Miguel, G. Cardona, Wearing comfort and blinking frequency with large diameter RGP contact lenses, Cont Lens Anterior Eye 35(Supp 81) (2012) E27. [26] D. Williams-Lyn, K. MacNeill, D. Fonn, The effect of rigid lens back optic zone radius and diameter changes on comfort, International Contact Lens Clinic 20(11-12) (1993) 223-229.

[27] D. Fadel, The influence of limbal and scleral shape on scleral lens design, Cont Lens Anterior Eye (2018).

298 [28] S.J. Vincent, D. Fadel, Optical considerations for scleral contact lenses: A review, Cont Lens 299 Anterior Eye 42(6) (2019) 598-613. [29] F. Berjandy, P. Nabovati, H. Hashemi, A. Yekta, H. Ostadimoghaddam, S. Sardari, M. Khabazkhoob, Predicting initial base curve of the rigid contact lenses according to Javal keratometry findings in patients with keratoconus, Cont Lens Anterior Eye (2020).

303 [30] J.C. Montalt, E. Porcar, E. Espana-Gregori, C. Peris-Martinez, Corneoscleral contact lenses for 304 visual rehabilitation after keratoplasty surgery, Cont Lens Anterior Eye (2020). 